\title{
Non Destructive Testing using the SQUID with Integrated Gradiometer
}

\author{
H. Nakane*, H. Adachi*, M. Walker, and U. Klein \\ *Department. of Electrical and Electronic Engineering, Muroran Institute of Technology, \\ Muroran 050, JAPAN \\ Department of Physics and Applied Physics, University of Strathclyde, Glasgow G4 ONG, UK
}

\begin{abstract}
Summary
We have used the integrated SQUID gradiometer in an unshielded environment to make eddy current nondestructive testing measurement on a multi-layer aluminum structure. The sensor consists of a niobium dc superconducting quantum interference device (SQUID) and a first-order gradiometer pick up coil on the same substrate. As a demonstration of their capabilities, subsurface defects in a multilayer aluminum structure have been located and mapped using eddy current with no magnetic shielding around the specimen or cryostat.
\end{abstract}

KEY WORDS: eddy current nondestructive testing, SQUID, integrated SQUID gradiometer, unshielded environment, multi-layer structure

\section{INTRODUCTION}

Conventional nondestructive testing is a well established technique that is used routinely to locate and characterize flaws in a various structures, such as aerospace, marine, nuclear and high-pressure vessels. One of the outstanding application in nondestructive testing is the reliable detection of deep sub-surface flaws that may be caused by corrosion and fatigue in multi-layer structure. This is a particular problem in nondestructive testing of aircraft where a method is being to detect flaws that occur in layers of aluminum around rivets without the removal of paint or disassembly of the part [1].

SQUIDs combined with a gradiometer are capable of detecting very nearby signals in the presence of much larger background noise from more distant sources. Although, many reseachers have used SQUID gradiometers to the nondestructive testing of aircraft and other structural systems and materials, most of them were done in the magnetic shield room to reject the background noise [2][3].

In this report, we have made an eddy current nondestructive testing of a multi-layer aluminum structure using the integrated SQUID gradiometer in an unshielded environment.

\section{EXPERIMENTAL PROCEDURE}

The nondestructive testing system consists of the integrated SQUID gradiometer in a fiberglass dewar and magnetically quiet $x-y$ scanning system. The dc SQUID is made by standard $\mathrm{Nb} / \mathrm{Al}-\mathrm{Al}_{2} \mathrm{O}_{3} / \mathrm{Nb}$ trilayer process[4], and has a double washer structure which avoid spatially uniform fields and interference. The intrinsic noise power spectral density of the bare SQUID is $4 \mu \Phi_{0} \mathrm{~Hz}^{-1 / 2}$ above $5 \mathrm{~Hz}$ [5]. The integrated planar gradiometer is an asymmetrical one fabricated with an all-niobium process on the same $\mathrm{Si}$ substrate of the SQUID [5][6]. The precision with which this can be done means that very high intrinsic balance and excellent device to device repeatability are possible. Although an integrated gradiometer has many advantages there is a still problem. It is that the SQUID acts as a magnetic anomaly for the nearby gradiometer. An asymmetric design is adopted to reduce the magnetic anomaly effects of the SQUID. Fig. 1 shows schematic 
layout of an asymmetric first-order planar gradiometer device. Its pick-up coils occupy an area of $2 \times 0.3 \mathrm{~cm}^{2}$, and has crossovers at 1.3 and $11.3 \mathrm{~mm}$. This gradiometer responds only to the first-order field gradient $\mathrm{dBx} / \mathrm{dz}$ and higher order gradients, with theoretically not respond to a uniform field in any direction. Fig. 2 shows the spatial response of the integrated planar gradiometer to a magnetic dipole. The source was a 1000-turn coil $18.2 \mathrm{~mm}$ long with a diameter of $5.8 \mathrm{~mm}$, and was directed to $\mathrm{x}$ direction. An ac current of $100 \mathrm{~mA}$ at $15 \mathrm{~Hz}$ was applied to it and the coil was moved over a distance of almost $30 \mathrm{~cm}$ directly beneath the grodiometer. In the region beyond $10 \mathrm{~cm}(\log x=1)$, theresponse has a nearly $\mathrm{x}^{-4}$ characteristic caused by a combination of the source field decay and the $x^{-1}$

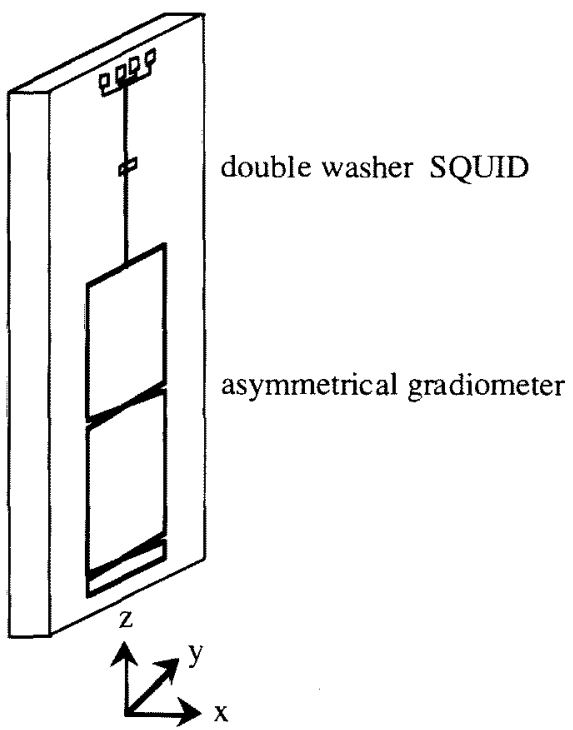

Fig. 1. Schematic layout of an asymmetric first-order planar gradiometer device.

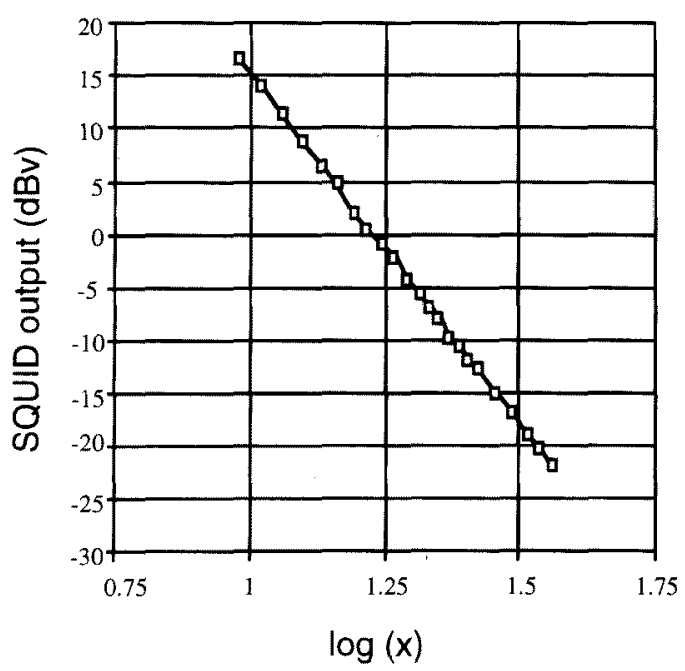

Fig. 2. The spatial response of the first-order gradiometerto a magnetic dipole. $(\log x=1$ at $x=10 \mathrm{~cm})$ characteristic expected of a first-order gradiometer. The fact that the spatial response does not level off at distances up to $30 \mathrm{~cm}$ indicates that the intrinsic balance of the gradiometer is better than one part in $10^{4}$. Fig. 3 shows the geometry of the scanning system. The integrated SQUID gradiometer device has been operated in a 1.5 litter fiberglass dewar with a hold time of over 24 hours. Minimum spacing of dewar's tail is $6 \mathrm{~mm}$. The integrated SQUID gradiometer is held vertically and is operated in a conventional flux-locked loop with a modulation frequency of $100 \mathrm{kHz}$. The gradiometer device itself has no magnetic shield, though an $18 \mu \mathrm{m}$ layer of aluminum foil is wrapped around the dewar to reduce $\mathrm{rf}$ interference. The dewar is suspended on a wooden frame above a computer controlled, magnetically quiet $x-y$ scanning system [7]. This system is located in a

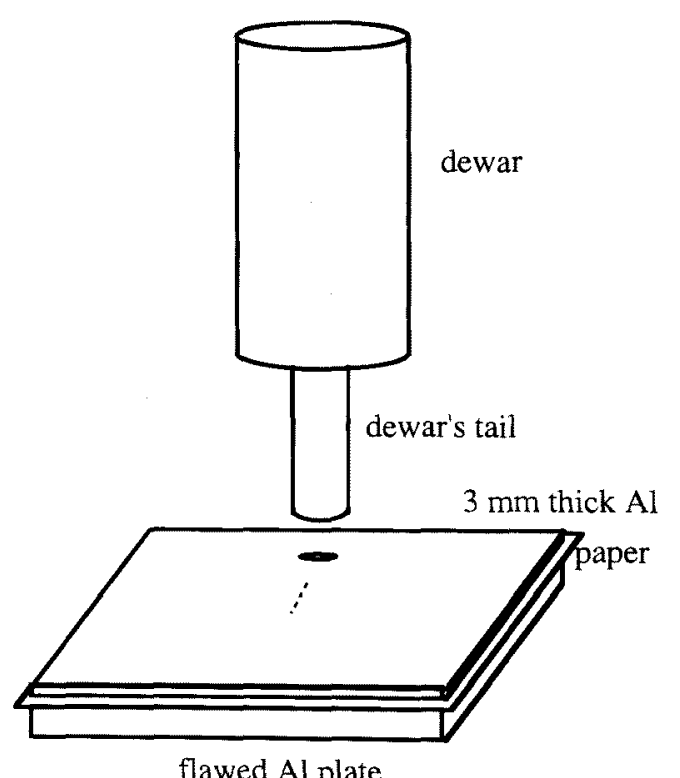

(a) the geometry of the scanning system

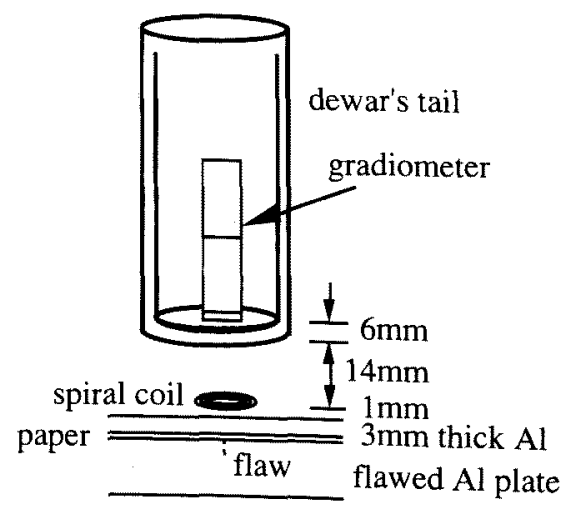

(b) cross sectional model

Fig.3. The geometry of the scanning system and a cross sectional model. 
conventional research laboratory, with nearby computers, power wiring and other disturbances. Eddy currents were induced in the test sample by a 4 turn, $4.5 \mathrm{~mm}$ diameter spiral copper coil etched onto a printed circuit board. The spiral coil was positioned $1 \mathrm{~mm}$ above the sample and 20 $\mathrm{mm}$ below the lower edge of the gradiometer. A center of the spiral coil was coincided with a central axis of the gradiometer in $\mathrm{z}$ direction. When the eddy current was induced in an uniform test sample, a symmetrical magnetic field with respect to the central axis of the gradiometer was generated, and the output signal of the gradiometer would be very small. If there was a flaw in the test sample, an asymmetrical magnetic field was generated, and detected with SQUID. A minimum size of a flaw which can be detected with the gradiometer should be increased with a size of gradiometer in y direction, and decreased with a distance between the sample and the gradiometer. Using a small spiral coil close to the sample under test enhances the spatial resolution of the measurement even though the sample-to-sensor lift-off distance is rather large, because a magnetic field distribution induced with the spiral coil becomes smaller. Since the flaw was about $4 \mathrm{~mm}$ from the coil, the $4.5 \mathrm{~mm}$ diameter of the coil is close to the optimum. A subsurface flaw's depth detected with the gradiometer was limited with a skin depth of the cover layer of the test sample. An eddy current at $70 \mathrm{~Hz}$ was applied to the coil. The conductivity of aluminum at this frequency is 18.1 MSm-1, giving a skin depth of $14.1 \mathrm{~mm}$.

\section{RESULTS AND DISCUSSION}

The system has been used to scan multi-layer aluminum samples (U.S. aircraft grade Al 7075-T651). As an example we have examined a plate $13 \mathrm{~mm}$ thick with a machined flaw $0.15 \mathrm{~mm}$ wide, $3 \mathrm{~mm}$ deep, and about $20 \mathrm{~mm}$ long on its top surface, covered with a $90 \mu \mathrm{m}$ layer of paper and then with a $3 \mathrm{~mm}$ layer of aluminum. The sensor was scanned at a speed of $2 \mathrm{~mm} / \mathrm{s}$ giving one measurement every $0.4 \mathrm{~mm}$. A magnetic field signal sensed by the gradiometer was lock-in detected with a reference of the coil current. A $550 \mathrm{~mA}$ rms current at $70 \mathrm{~Hz}$ was applied to the coil, giving a vertical magnetic field of $0.8 \mathrm{mT}$ at the sample surface. The resulting 2D image of the unprocessed SQUID output signal is shown in Fig. 4(a), which is a mapping results of the detected magnetic field strength. In order to reveal a polarity of themagnetic field, it was shown with a gray scale, background gray is a zero level, black is a minus level, white is a plus level. When the eddy current was induced in an uniform test sample, a symmetrical magnetic field with respect to the central axis of the gradiometer was generated, and the output signal of the gradiometer would be very small. If there was a flaw in the test sample, an asymmetrical magnetic field ( $\mu \mathrm{T}$ order) was generated, and detected with the gradiometer. At the end-point of the flaw, the detected signal became maximum, because an asymmetry of the magnetic field distribution

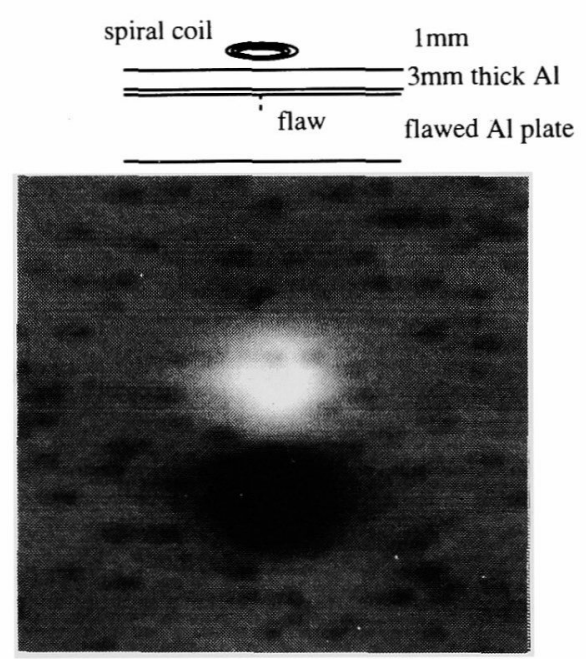

(b) A 2D scan of a single-layer aluminumsample.

The scanning area is $85 \times 80 \mathrm{~mm}$.

Fig. 4. 2D scans of a multi-layer aluminum sample and a single-layer aluminum sample.

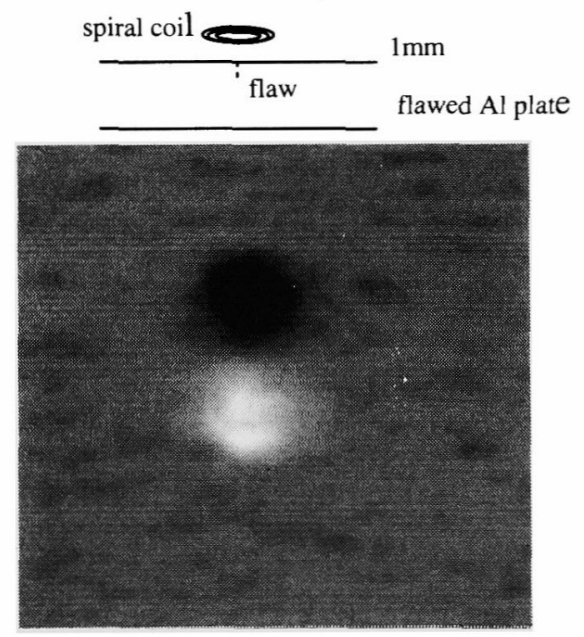

(b) A 2D scan of a single-layer aluminumsample. The scanning area is $85 \times 80 \mathrm{~mm}$.

Fig. 4. 2D scans of a multi-layer aluminum sample and a single-layer aluminum sample. 
became maximum. These corresponds centers of a black circle and a white circle patterns in Fig.4(a). At the black pattern and the white pattern, directions of magnetic field are opposite. A length between the centers of black and white patterns corresponds a length of the flaw.

There are frequent bursts of $\mathrm{rf}$ detected by the SQUID as equipments in the building are switched on and off. The bursts of rf are showed as black dots in Fig. 4(a). Occasionally this impulse noise was severe to unlock the SQUID. The 2D scanning image with single layer sample is also shown in Fig. 4(b). In this case, a $277 \mathrm{~mA} \mathrm{rma}$ current at $70 \mathrm{~Hz}$ was applied to the coil, giving a magnetic field of $0.4 \mathrm{mT}$ at the sample surface. A length of the flaw estimated with Fig. 4(a) is same length with Fig. 4(b).

\section{CONCLUSIONS}

Low-temperature superconductor thin-film integrated SQUID gradiometer have been fabricated using wellestablished standard niobium trilayer technology. Firstorder grdiometric devices have been mounted in a small, nonmagnetic fiberglass dewar and we have demonstrated that they can be operated in an unshielded laboratory environment. Our gradiometric magnetic field sensors have been used for exploratory eddy current nondestructive testing on a $2 \mathrm{D}$ scanning rig.

Their performance has been found more than adequate to locate flaws in a multi-layer aluminum sample which simulate the defects in aircraft.

\section{ACKNOWLEDGMENT}

We are indebted to Dr. A. Cochran for fruitful discussion. The integrated SQUID gradiometer device were fabricated by R.G. Weston.

(Manuscript received April 7, 1997, revised Aug. 18, 1997)

\section{REFERENCES}

[1]. Dixon T. E., Silk M. G. and MacKeith D. J. , INSIGHT, $36, \mathrm{p} 256,1994$

[2]. Wikswo Jr. J. P. , IEEE Trans. Appl. Sup., 5, p74,1995

[3]. Chinone K., Nakayama S., Morooka T., Odawara A. and Ikeda M.,IEEE Trans. Appl. Sup., 7, p3271,1997

[4]. Hutson D., Weston R. G., Bain R. J. P., Lawrenson M. J.,Pegrum C. M., Popova K. and Scarfone R. IEEE Trans
Appl. Supercond., 6, p2295,1995

[5]. Klein U., Walker M. E., Cochran A., Hotson D., Lang D., Weston R. G. and Pegrum C. M. , Supercond. Sci. Technol., 9, p1 ,1996

[6]. Walker M. E., Nakane H., Cochran A., Weston R. G., Klein U. and Pegrum C. M. , Appl. Phys. Lett., 71,p131,1997

[7]. Cochran A., Macfarlane J. C., Morgan L. N. C., Kuznik J., Weston R. G., Hao L., Bowman R. M. and Donaldson G.B. ,IEEE Trans Appl. Supercond., 4, p128,1994

Hideaki Nakane (nonmember) received the B.S. degree in

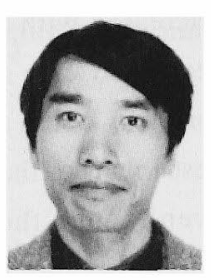
1976, M.S. degree in 1978 in electrical engineering, and D.E. degree in electrical engineering from Hokkaido University in 1981. He joined Central Research Laboratory, Hitachi Ltd. , where he worked on superconductive devices. He moved to Muroran Institute of Technology as an associate professor in 1991. His current interest is an application of superconductive devices. He also engaged in reseach on surface physics.

Hiroshi Adachi (member) received a B.S. degree in 1965,

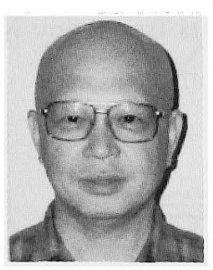
a M.S. degree in 1967 and a doctor of engineering in 1971 from Tohoku University. He joined to Tohoku University as a research associate in 1971. He moved to Muroran Institute of Technology as a associate professor in 1981. In 1991, he promoted to a professor. He has engaged in reteach on materials. He also engaged in research on sensors, vacuum technology and surface physics.

Morag Walker (nonmember) graduated with a BSc in

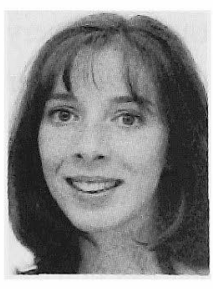
Applied Physics from the University of Strathclyde in Glasgow, Scotland in 1994 and with a PhD in the Design of Planar Integrated SQUID Gradiometers for Nondestructive Evaluation in 1997, also from the

University of Strathclyde. She is currently working as a sonar systems engineer with Thomson Marconi Sonar Limited. 
Udo Klein (nonmember) was born in Andernach, Germany,

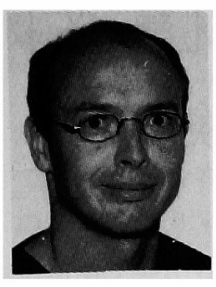

on January 2, 1960. He received the Dipl.-Ing. and Dr.-Ing. degrees in Electroncis Engineering from the Technical University Braunschweig, Germany, in 1986 and 1990,respectively. In 1987 he joined the Institute of HighFrequency Engineering, Technical University Braunschweig, as a Research Engineer and Assistant Lecturer. Between 1990 and 1993 he was a Guest Researcher at the Electrotechnical Laboratory, Tsukuba, Japan, where he worked on Josephson voltage standard systems. He then worked in the Superconductivity Research Group, University of Birmingham, England, on high-Tc superconductor thin films and devices. In 1995 he joined the Superconducting Devices Research Group, University of Strathclyde, Scotland, where he worked on SQUID systems for nondestructive testing. During 1996 he visited the Department of Materials and Production Engineering, University of Naples, Italy, for 5 months as a consultant for the design of an eddy current SQUID NDE system. He is currently with the Department of Physics at the Technical University of Denmark, working on high-Tc flux-flow devices.

Dr. Klein is a member of the VDI, the IEEE, and the IoP. 